\title{
SPONTANEOUS PERFORATION OF THE COLON IN THE NEWBORN INFANT
}

\author{
BY \\ S. E. LEVIN and C. ISAACSON \\ From the Department of Paediatrics, Baragwanath Hospital, and \\ the South African Institute for Medical Research, Johannesburg
}

(RECEIVED FOR PUBLICATION SEPTEMBER 15, 1959)

If one excludes those cases of perforation which are secondary to volvulus, intestinal atresia, congenital bands, intussusception, herniation of the bowel, meconium ileus or Hirschsprung's disease, there remains a group of newborn infants who develop perforation of the colon without apparent cause.

Spontaneous perforation of the colon in the neonate was first described by Breslau in 1863 . It is a rare event and up until 1939 only 26 case reports could be traced in the medical literature by Thelander.

A number of factors have been considered in the aetiology of spontaneous perforation such as trauma during delivery (Zillner, 1884), congenital defects of the bowel with diverticulum formation (Fischer, 1928), localized vascular insufficiency, and intrauterine or post-partum infection (Thelander, 1939). In 1888 Paltauf reported five cases which he attributed to coprostasis. This aetiological concept has recently received support from the clinico-surgical and pathological studies by Zachary (1957) and Emery (1957) of a group of newborn infants in whom inspissated faecal plugs gave rise to stercoral ulceration with perforation. These authors suggest that the observed faecal impaction may be due to an imbalance between fluid absorption and of propulsion of material along the colon. More recently Haas (1958) has reported a newborn infant with perforation of the transverse colon in whom a haemangiomatous abnormality at the site of perforation was demonstrated.

There still remains a group of newborn infants with one or more perforations of the colon and in whom no clear cut cause can be discovered.

In this paper two further cases of spontaneous perforation of the colon are reported. In one of these, perforation occurred during or immediately after birth and this was associated with marked intraperitoneal haemorrhage. The cause of the perforation was undetermined. The other child was a premature infant who developed the perforation nine days after delivery and histological examination showed fungal infection at the site of perforation.

\section{Case Reports}

Case 1. This female infant was delivered at term as a vertex presentation by a district midwife after an uneventful pregnancy. The child appeared to be vigorous after birth, but abdominal distension with oedema of the vulva was noted. One blood-stained stool was passed after birth and this led to the admission of the child to this hospital.

On examination, six hours after delivery, the child appeared somewhat pale but not greatly distressed. The weight was $5 \mathrm{lb} .5 \mathrm{oz}$. and the rectal temperature was $95^{\circ} \mathrm{F}$. The most striking feature was marked distension of the abdomen. There were prominent dilated veins on the anterior abdominal wall which was oedematous in its lower half. There was also oedema of the vulva. Percussion of the abdomen elicited shifting dullness. A radiograph of the abdomen showed air in the stomach and small bowel. There were no fluid levels indicating obstruction. Examination of venous blood revealed a haemoglobin level of $9.0 \mathrm{~g}$./ $100 \mathrm{ml}$.

An abdominal paracentesis at McBurney's point yielded blood-stained fluid with a haemoglobin level of $2.5 \mathrm{~g} . / 100 \mathrm{ml}$. A preoperative diagnosis of rupture of the liver was made and the child was transfused with $100 \mathrm{ml}$. of group $\mathrm{O} \mathrm{Rh}$ positive compatible blood. At laparotomy blood-stained fluid escaped from the peritoneal cavity. Floating in this fluid were fragments of fresh meconium (later confirmed histologically). Careful inspection of small and large bowel failed to reveal a perforation. A Meckel's diverticulum was found, but it appeared to be intact. No rupture of the liver was found. The omentum was stained green by the meconium but there was no evidence of peritonitis.

The abdomen was closed again and the child was maintained on intravenous fluid $(0 \cdot 2 \%$ saline in $5 \%$ invert sugar) while gastric suction was applied. There was no indication of postoperative bleeding, but 
48 hours after the operation she suddenly collapsed and died.

At necropsy the peritoneal cavity contained a small quantity of semi-fluid dark brown material. There was some adherence of the bowel loops. A small perforation 3-4 mm. in diameter was found in the region of the hepatic flexure of the ascending colon and a small quantity of meconium was emerging from the perforation. The anatomical position of the bowel at autopsy appeared normal and there was no evidence of volvulus or intussusception. There was thus no obvious cause for the perforation. Other organs showed no abnormalities except for some patches of atelectasis of the lungs.

Histology of sections of the bowel at the site of perforation revealed merely a break in the continuity of the wall with some necrosis of the wall adjacent to the perforation. Ganglion cells were present in normal numbers. The pancreas showed no obvious lesion. The lungs were congested and oedematous.

Case 2. This male infant was born at this hospital weighing $3 \mathrm{lb}$. The mother developed puerperal sepsis and the infant at 3 days old was transferred to the premature infant unit, his weight then being $2 \mathrm{lb}$. $11 \frac{1}{2} \mathrm{oz}$. Apart from mild icterus, no abnormalities were detected

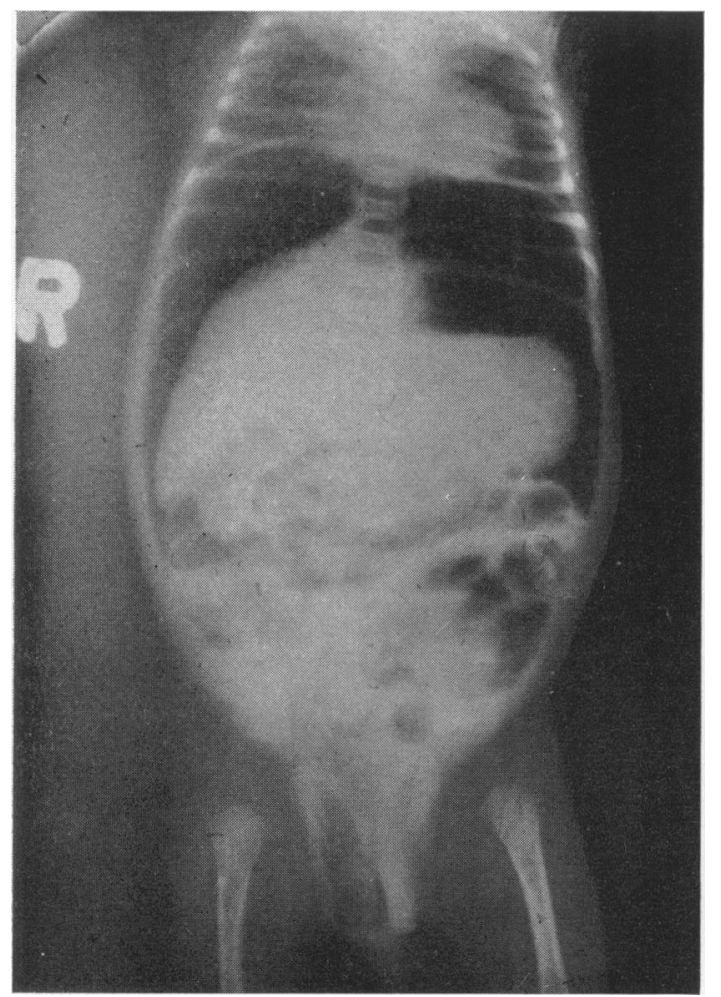

FIG. 1.-Radiograph of the abdomen showing a large accumulation of air under the diaphragm and in the right scrotal sac. on clinical examination. He was given feeds of undiluted expressed breast milk from the third day. On the fifth day of life the jaundice had become more intense. The serum bilirubin level was $12 \mathrm{mg} . / 100 \mathrm{ml}$. Meconium was passed on the fourth and sixth days of life but no stools were noted on the seventh, eighth and ninth days of life. On the ninth day, the infant vomited a feed which was not stained with bile. Some slight distension of the abdomen was noted. A glycerine suppository was prescribed and oral feeding of expressed breast milk was continued. Later in the day two brown watery stools were passed and one of them was bloodstained.

The following day (day 10) the infant continued to vomit and developed gross gaseous distension of the abdomen. The clinical state of hydration was satisfactory. A radiograph of the abdomen revealed the presence of a well-marked pneumoperitoneum (Fig. 1). Gas was present in the small bowel without evidence of fluid levels, and for this reason the site of perforation was thought to be in the colon. Feeds were stopped and intravenous therapy was started with $0.2 \%$ saline in $5 \%$ invert sugar with the addition of $2.0 \mathrm{~g}$. of potassium chloride to $1,000 \mathrm{ml}$. of solution. The child was also given 100,000 units of penicillin intramuscularly every eight hours and $50 \mathrm{mg}$. of streptomycin intramuscularly every 12 hours. On the eleventh day the child's abdomen was still markedly distended and there was oedema of the scrotum. An ill-defined mass could be palpated in the left flank. The infant had also developed oral thrush which was treated with 50,000 units of Nystatin ('mycostatin') every eight hours. On the tenth day the child passed two small scybala. The child's condition continued to deteriorate and death took place on the twelfth day of life.

At necropsy there was marked meconium peritonitis. The peritoneal cavity contained $150 \mathrm{ml}$. of

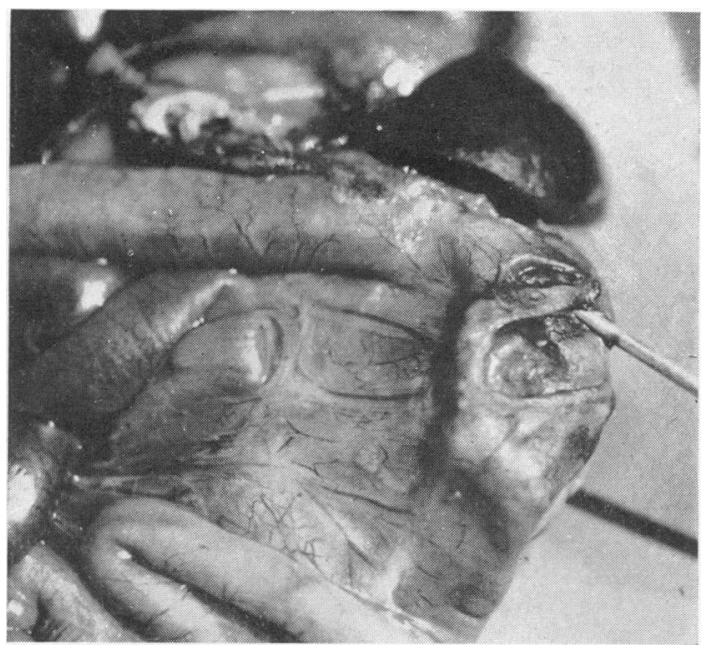

FIG. 2.-Colon with large perforation in anterior wall immediately distal to splenic fiexure. 


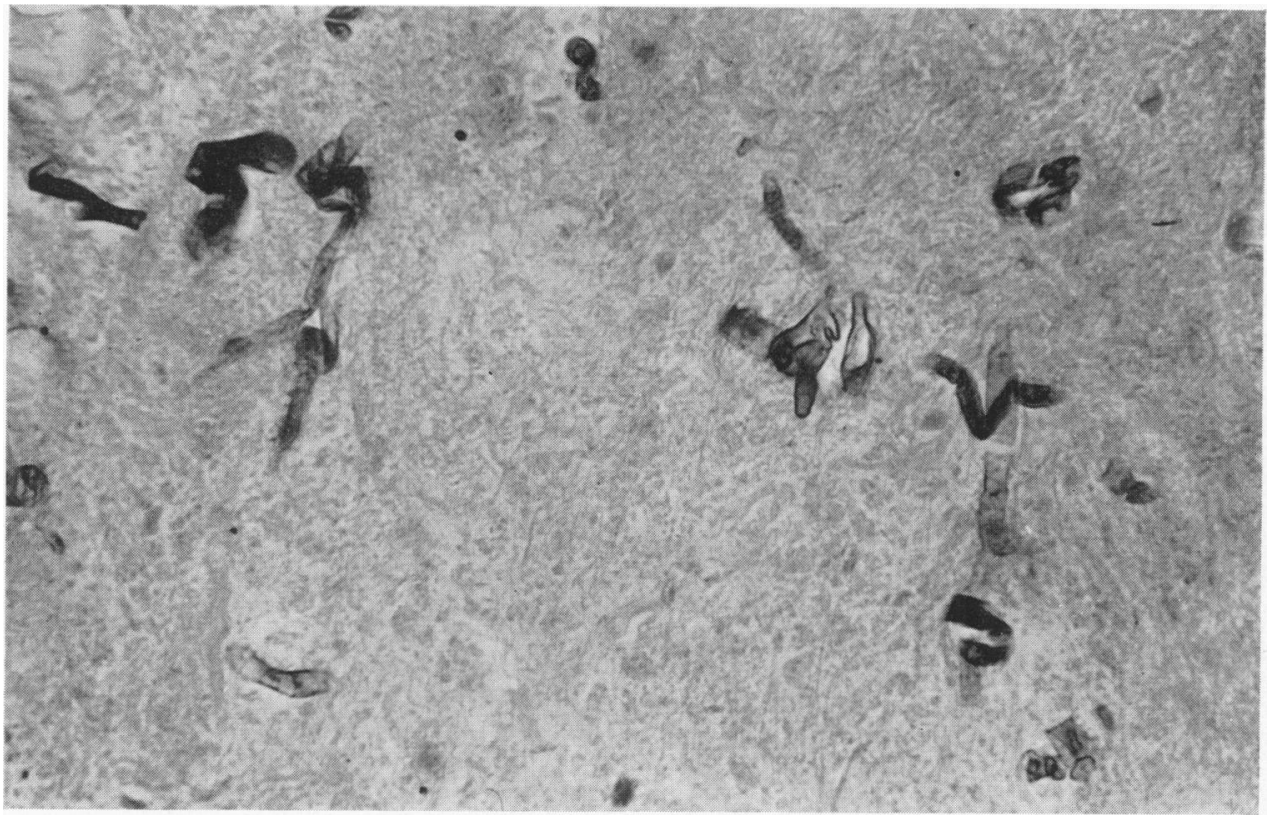

Fig. 3.-Wall of colon adjacent to the perforation showing numerous fungal hyphae.

(Periodic-acid Schiff $\times$ 480.)

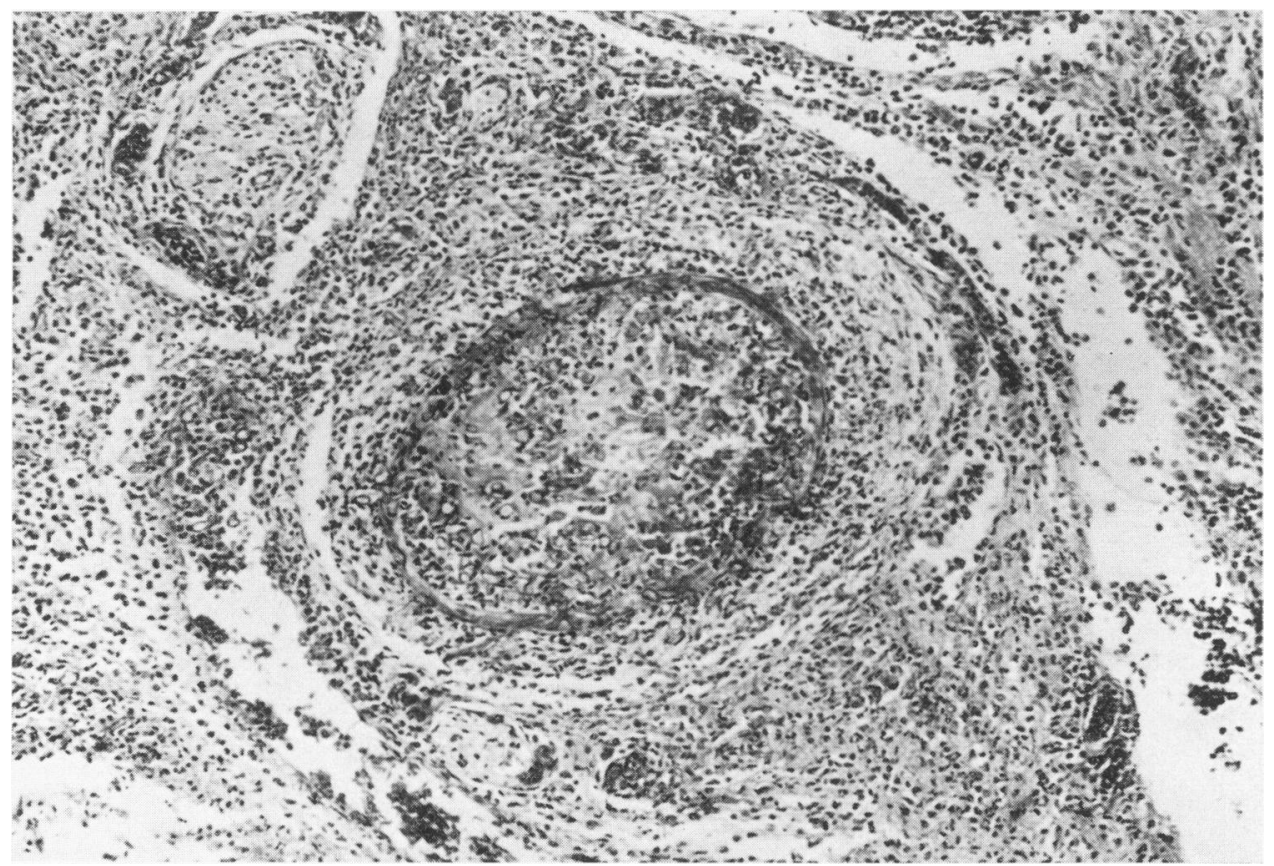

FIG. 4.-Blood vessel in wall of colon adjacent to the perforation showing invasion by inflammatory cells and fungal hyphae. (Haematoxylin and eosin $\times 150$.) 
green purulent fluid. Loops of bowel were densely adherent to the lateral abdominal wall on the left. A large perforation $0.5 \mathrm{~cm}$. in diameter (Fig. 2) was present about $2.5 \mathrm{~cm}$. below the splenic flexure in the anterior wall of the descending colon.

There were still some yellow flecks of inspissated meconium around the perforation and floating in the peritoneal exudate. Anatomically the bowel was normal, and there was no evidence of a volvulus or an intussusception.

Section of the bowel wall at the site of the perforation showed the surrounding mucosa to be necrotic and haemorrhagic. There was haemorrhage into the submucosa and infiltration of the entire wall by polymorphs. The serosa was acutely inflamed and covered by necrotic debris. Fungal hyphae consistent in appearance with the fungus mucor, or the related rhizopus, were present in the lumen of the bowel, in the wall and in the serosa (Fig. 3). The fungal filaments were large and showed absence of cross-wall formation with free and irregular branching, features suggesting origin from the mucoraceae. In many areas the fungi were invading the walls of blood vessels and had come to lie within their lumens (Fig. 4). Unfortunately cultures of the fungus were not made before the tissues were fixed in formalin.

Histology of the pancreas was normal.

\section{Discussion}

Our first case presented the clinical picture of intraperitoneal haemorrhage. Hence a preoperative diagnosis was made of rupture of the liver, which is the commonest solid viscus to be damaged in the newborn infant (Stein and Wright, 1953; Arden, 1951; Brown, 1957).

At operation the surprise finding of meconium in the peritoneal cavity, in addition to the blood, indicated perforation of a hollow viscus. The site of perforation was not detected at laparotomy. This difficulty of locating the site of perforation has been encountered by others, and it happened in four of the cases reported by Thelander (1939). Standard (1952) in reporting his case, which was successfully treated surgically, admits that the perforation would not have been visible but for the presence of leaking barium suspension which he had given to the infant. In a case reported by Franklin and Hosford (1952), the perforation was only found at the second operation.

Necropsy in our first case failed to reveal a cause for the perforation, but it must be admitted that a close study of the blood supply to the affected segment of bowel was not carried out. The clinical and experimental studies of Louw and Barnard (1955) have indicated that vascular occlusion plays an important role in the production of intestinal atresia in the newborn infant. Their work may have some application in the elucidation of the aetiology of spontaneous perforation of the colon. We suggest that, in future, a careful study be made of the blood supply to the area of a spontaneous perforation of the colon. We have assumed that the intraperitoneal bleeding in our case arose from the same site as the perforation, but this was not obvious at autopsy, the bleeding having ceased postoperatively.

Case 2 is of interest in that this premature infant was progressing satisfactorily until the ninth day of life. The child was starting to regain its postnatal weight loss, when it failed to pass stools for three successive days. As in Case 1 there was the passage of one blood-stained stool and it is possible that this finding in the presence of abdominal distension may be of help in the diagnosis of spontaneous perforation of the bowel.

Three possibilities suggest themselves as to the cause of the perforation in Case 2. Firstly this perforation could have followed on stercoral ulceration caused by inspissated meconium. The passage of two small hard pieces of faeces and the finding of residual thickened meconium at necropsy are in favour of this suggestion. However, we were unable to analyse the water content of the meconium as was done by Emery (1957).

Secondly the perforation may be aetiologically connected with the finding of mucor (or rhizopus) in the bowel wall at the site of perforation. Mucor has been found in association with perforation in other parts of the gastro-intestinal tract. Moore, Anderson and Everett (1949) described a widespread ulcerative colitis in a non-diabetic female aged 37 years caused by mucor which resulted in death after perforation and generalized peritonitis. Watson (1957) reported perforation of the stomach in association with mucormycosis in a child of 26 months suffering from malnutrition. A similar perforation was described by Gatling (1959) in a 4-day-old neonate. Thus this fungal infection may have been the primary cause of the perforation in Case 2. It is also possible that the fungus aggravated a perforation caused initially by hardened meconium.

Until recently the prognosis of spontaneous perforation was thought to be uniformly hopeless. However, in 1952, Standard, in America, and Franklin and Hosford, in Britain, reported survivals following surgical repair. Thus laparotomy is probably advisable whenever the condition of the patient permits. Preliminary decompression of the abdomen may be necessary to increase vital capacity (Standard, 1952). 


\section{Summary}

Two cases of spontaneous perforation of the colon are reported, one in a full term and the other in a premature infant. There was an associated intraperitoneal haemorrhage in Case 1, and in this infant no demonstrable cause was found for the perforation.

In the second baby the perforation was found in association with inspissated meconium and the presence of fungal infection in the bowel wall morphologically either mucor or rhizopus. Unfortunately cultures of the organisms were not taken.

Our thanks are due to Dr. I. Frack, Superintendent, Baragwanath Hospital, for permission to publish these cases, Dr. E. Kahn, Senior Paediatrician, for criticism and advice, the Director of the South African Institute for Medical Research, for facilities granted, and Mr. M. Ulrich for the photographs.

\section{REFERENCES}

Arden, F. (1951). Rupture of the liver in the newborn. Med. J. Aust., 2, 632 .
Breslau (1863). Cited by Thelander (1939).

Brown, J. J. M. (1957) Hepatic haemorrhage in the newborn. Arch. Dis. Childh., 32, 480.

Emery, J. L. (1957). Abnormalities in meconium of the foetus and newborn. Ibid., 32, 17.

Fischer, A. E. (1928). Fetal peritonitis; report of a case following spontaneous rupture of the large intestine. Amer. J. Dis. Child., 36, 774 .

Franklin, A. W. and Hosford, J. P. (1952). Meconium peritonitis due to a hole in the foetal intestinal wall and without obstruction. Brit. med. J., 2,257 .

Gatling, R. R. (1959). Gastric mucormycosis in a newborn infant a case report. A.M.A. Arch. Path., 67, 249.

Haas, L. (1958). Neonatal perforation of the colon. Arch. Dis. Childh., 33, 362.

Louw, J. H. and Barnard, C. N. (1955). Congenital intestinal atresia ; observations on its origin. Lancet, $2,1065$.

Moore, M., Anderson, W. A. D. and Everett, H. H. (1949). Mucormycosis of the large bowel. Amer. J. Path., 25, 559.

Paltauf, A. (1888). Die spontane Dickdarmruptur der Neugebornen. Virchows Arch. path. Anat., 111, 461.

Standard, J. E. (1952). Perforation of colon in the newborn infant recovery following operation. Amer. J. Surg., 83, 107.

Stein, F. E and Wright, L. T. (1953). Rupture of hollow and solid viscera in the newborn; an analysis of twenty-five cases. Harlem Hosp. Bull., 6, 32. (Year Book of Paediatrics, 1954-1955, p. 26.)

Thelander, H. E. (1939). Perforation of the gastrointestinal tract of the newborn infant. Amer. J. Dis. Child., 58, 371.

Watson, K. C. (1957). Gastric perforation due to the fungus Mucor in a child with kwashiorkor. S. Afr. med. J., 31, 99.

Zachary, R. B. (1957). Meconium and faecal plugs in the newborn. Arch. Dis. Childh., 32, 22.

Zillner, E. (1884). Ruptura flexurae sigmoidis neonati inter partum. Virchows Arch. path. Anat., 96, 307. 